\title{
A Settlement Landscape Reconstruction Approach Using GIS Analysis with Integrated Terrain Data of Land and Water: A Case Study of the Panlongcheng Site in the Shang Dynasty (Wuhan, China)
}

\author{
Jianfeng Liu ${ }^{1,2}$, Qiushi Zou ${ }^{3,4, *}$, Qingwu Hu ${ }^{1}$, and Changping Zhang ${ }^{3,4}$ \\ 1 School of Remote Sensing and Information Engineering, Wuhan University, Wuhan 430079, China; \\ jianfengliu@whu.edu.cn (J.L.); huqw@whu.edu.cn (Q.H.) \\ 2 The State Key Laboratory of Information Engineering in Surveying, Mapping and Remote Sensing, \\ Wuhan University, Wuhan 430079, China \\ 3 School of History, Wuhan University, Wuhan 430079, China; 00009171@whu.edu.cn \\ 4 Archaeological Institute for Yangtze Civilization, Wuhan University, Wuhan 430072, China \\ * Correspondence: zouqiushi@whu.edu.cn; Tel.: +86-1770-272-3997
}

\section{check for}

updates

Citation: Liu, J.; Zou, Q.; Hu, Q.; Zhang, C. A Settlement Landscape Reconstruction Approach Using GIS Analysis with Integrated Terrain Data of Land and Water: A Case Study of the Panlongcheng Site in the Shang Dynasty (Wuhan, China). Remote Sens. 2021, 13, 5087. https:// doi.org/10.3390/rs13245087

Academic Editors: Bruno Fabio and Maria Geraga

Received: 27 October 2021

Accepted: 7 December 2021

Published: 14 December 2021

Publisher's Note: MDPI stays neutral with regard to jurisdictional claims in published maps and institutional affiliations.

Copyright: (c) 2021 by the authors. Licensee MDPI, Basel, Switzerland. This article is an open access article distributed under the terms and conditions of the Creative Commons Attribution (CC BY) license (https:/ / creativecommons.org/licenses/by/ $4.0 /)$.

\begin{abstract}
The landscape of ancient sites has changed greatly with the passage of time. Among all of the factors, human activities and the change in natural environment are the main factors leading to the change in site landscape. The Panlongcheng site, which is located in Hubei Province, China, has a history of 3500 years with the most abundant relics in the Yangtze River Basin during the Shang Dynasty. As a near-water site, the landscape of the Panlongcheng site is greatly affected by water level changes and water conservancy activities. In this paper, by using spatial information technology, the data obtained from land and underwater archaeological exploration were integrated to restore landscapes of Panlongcheng sites in different periods. After removing modern artificial features and topsoil, the landscapes of the sites before the Shang Dynasty, in the Shang Dynasty and modern time were reconstructed. Combining historical records of water level changes, the landscape and water-land distribution of the Panlongcheng site were compared. The analysis results reflect the interaction between water level changes and human activities in this region for thousands of years, and support the archaeological findings in the near-water area of the Panlongcheng site, which provides a new idea for the landscape reconstruction and analysis of near-water sites.
\end{abstract}

Keywords: settlement landscape; water-land distribution; Panlongcheng site; environment changes; human activities

\section{Introduction}

With the development of GIS and archaeological technology, geographic data are widely used to augment the exploration and analysis of past lifeways [1]. A wide variety of spatial data, such as climatic, geological and cultural, were combined to estimate how long-term interactions among these factors contribute to the changes in natural environment and anthropogenic landscapes [2]. Archaeological sites and relics can be displayed with $3 \mathrm{D}$ reconstruction and visualization technology. For example, the landscapes of sites are reconstructed with the GIS approach. Landscapes of different periods can help archaeologists fully understand the spatial distribution and evolution process of archaeological sites, which provides a new viewpoint for archaeologists to study the relationship between natural environment changes and human activities. Landscape archaeology is becoming increasingly important with the progress of GIS technology [3,4]. Landscapes in those sites near water vary a lot in different periods because of environment changes and human activities. The change in water level in rivers and lakes, the development of agriculture and fishery and the construction of water conservancy facilities will have a great impact on the landscape of near-water sites. 
The Panlongcheng site is located in the northeast edge of Jianghan Plain, which lies in the transitional zone between the Ta-pieh Mountains and the alluvial plain on the North Bank of the Yangtze River. The site is close to Panlong lake and Fu River. This paper took the Panlongcheng site as an example and proposed a new method to reconstruct the landscape of cultural sites near water and analyze water-land relationship of the site in different periods, which revealed the profound impact of human activities and environmental changes on the landscape of the archaeological site and provided a reference for analysis of human activities and archaeological work in sites near water.

The field archaeological work of the Panlongcheng site has lasted for more than 60 years. Through years of archaeological excavation, the archaeologists have formed a clear understanding of the cultural features of the site, the time of its rise and fall and the settlement layout [5]. However, for a long time, scholars have had a lack of understanding of the landscape and water-land distribution of the Panlongcheng site during the Shang culture period. On the one hand, the Panlongcheng site is close to rivers and lakes; therefore, water level changes have a profound impact on the living environment. In the past 60 years, archaeologists have found many tombs, bronzes, pottery and other important relics in the lakeshore and underwater in the Panlong lake [6]. Therefore, the geomorphic landscape of the Panlongcheng site in the Shang Dynasty may be quite different from the current situation due to changes in water level [7]. On the other hand, the Panlongcheng site is located on the low hills near the lake. From the middle and late Shang Dynasty to the present, human activities in different periods caused some significant changes to the landscape of the Panlongcheng site. For example, in the past 100 years, a series of activities such as land leveling, construction of the dike and excavation of fishponds were carried out by local villagers, which caused changes in the landscape of the Panlongcheng site [8].

To figure out the spatial distribution and evolution process of the site, it is very necessary to reconstruct landscape with water-land relationship of the site in different periods. From 2012 to 2016, systematic archaeological exploration in the land area of the Panlongcheng site was carried out, and the 3D coordinates and stratum information of each drill hole were recorded by GIS software [9]. Both underwater and land archaeological data are necessary to restore the landscape. With the improvement of underwater topography measurement, it is more realistic to reflect the underwater topography comprehensively and clearly, which makes underwater archaeology possible [10]. The underwater archaeology can be carried out with a multi-resolution morpho-bathymetric survey [11]. Combined with GIS-based reconstructions of lake level change, paleo-ecological modeling of the habitats of extant and extinct bovids are analyzed, indicating that human occupation of these sites coincided with substantial declines in the level of water [12].

At present, underwater archaeological survey methods can be divided into two categories: optical and acoustic methods. Among the optical methods, diving survey is the most common method. Divers use marking, grid and strip tools to survey underwater cultural relics. However, diving survey will be a very time-consuming and high-risk job in the complex underwater environment. Furthermore, the results were greatly influenced by subjective judgment of divers. Underwater photogrammetry provides a good solution for reaching more objective conclusions. By taking a series of photos from different angles, 3D models of underwater landscape can be restored [13]. Acoustic methods are widely used in underwater archaeology. Among them, multi beam sonar and side scan sonar are widely used to identify and demarcate underwater targets [14]. Experiments and archaeological investigations show that side scan sonar can distinguish organic and inorganic materials, and man-made and natural materials [15]. However, the images presented by side scan sonar are often distorted, which lacks three-dimensional information [16]. Multi beam sonar carries out high-precision real-time 3D imaging of underwater topography. For example, high-precision multi beam sonar is used to identify the sunken ships and reconstruct the underwater topography [17]. During the underwater investigation of the Danjiangkou Reservoir, the diving survey method was used to explore the ancient city of Junzhou. Moreover, multi beam sounding system, side sonar, shallow profiler, underwa- 
ter 3D scanner, GPS positioning system and other equipment and technology were used to explore underwater site and master the scale and preservation of the ancient city of Junzhou [18].

In order to figure out the distribution of underwater ancient ruins and restore the geographical landscape of the Panlongcheng site in the Shang Dynasty, underwater topographic measurement of Panlong Lake was carried out by using single beam ultrasonic sounder [19]. On this basis, underwater archaeological exploration was carried out. Cultural layers and pottery pieces of the Shang Dynasty were found $5.1 \mathrm{~m}$ below the lake surface (taking the highest water level of $22.6 \mathrm{~m}$ in the rainy season as reference) in this underwater exploration. Therefore, we can confirm that the water level of Panlong Lake in the Shang Dynasty was at least $5.1 \mathrm{~m}$ lower than modern time. At that time, water-land distribution and geomorphic landscape of the Panlongcheng site was greatly different from the present [19]. With the historical water level change, the landscape and water-land distribution in the Panlongcheng site are studied.

In this paper, both archaeological exploration data of land area and lakes are integrated to restore the geomorphic landscape of the Panlongcheng site. The water-land distribution of the Panlongcheng site in the Shang Dynasty and modern time is analyzed. Through a long period of landscape change analysis, the profound impact of human activities and environmental changes on the landscape of ancient sites is revealed, which provides a reference for analysis of human activities and archaeological work in sites near water.

\section{Materials and Methods}

\subsection{Study Area and Materials}

\subsubsection{Study Area}

The Panlongcheng site is located in the northern suburb of Wuhan, Hubei Province, China. During the Shang Dynasty (16th century BC-13th century BC), with the Central Plains culture expanding southward, it became the central city in the Yangtze River Basin, reflecting the development level of Chinese civilization in the Yangtze River Basin at that time. Since the 1960s, archaeologists have found abundant relics in the early Shang Dynasty in the Panlongcheng site, such as ramparts, moats, large-scale palace buildings, noble tombs and copper casting workshops [9]. Scholars generally believe that the Panlongcheng site was the most important city in the Yangtze River Basin during the early Shang Dynasty. Moreover, the Panlongcheng site may be closely related to the Shang Dynasty's control and plunder of copper resources in the Yangtze River Basin [5]. Location and satellite image of the Panlongcheng site are shown in Figure 1.

The Panlongcheng site covers $3.95 \mathrm{~km}^{2}$. Rivers, lakes and low hills crisscross in this area. The site is distributed along the Panlong lake and on a low hill to the north of Fu River. Landscape of the site is lower in the south and higher in the north, with an altitude of 19.5-37.4 m. Every year in the dry season (from December to March of the following year), the water level of Panlong lake and Fu River drops, and the ash pits and tombs of the Shang Dynasty can be exposed on the lakeshore. Since the 1980s, archaeologists have excavated several tombs at the edge of hillock near the lake such as Yangjiazui, Wangjiazui, Lijiazui and Xiaozui in the Panlongcheng site. The distribution of cultural sites is shown in Figure 2. These tombs were submerged by Panlong lake or Fu River in rainy season, but exposed in dry season. Therefore, archaeologists speculate that the water level of Panlong lake and Fu River in the Shang Dynasty should be lower than water level in the modern time. In other words, the lake basin and riverbed near the Panlongcheng site were probably exposed in the Shang Dynasty. Human activity space in the Panlongcheng site in the Shang Dynasty should have extended from the top of the hillock to the area submerged by water today. 


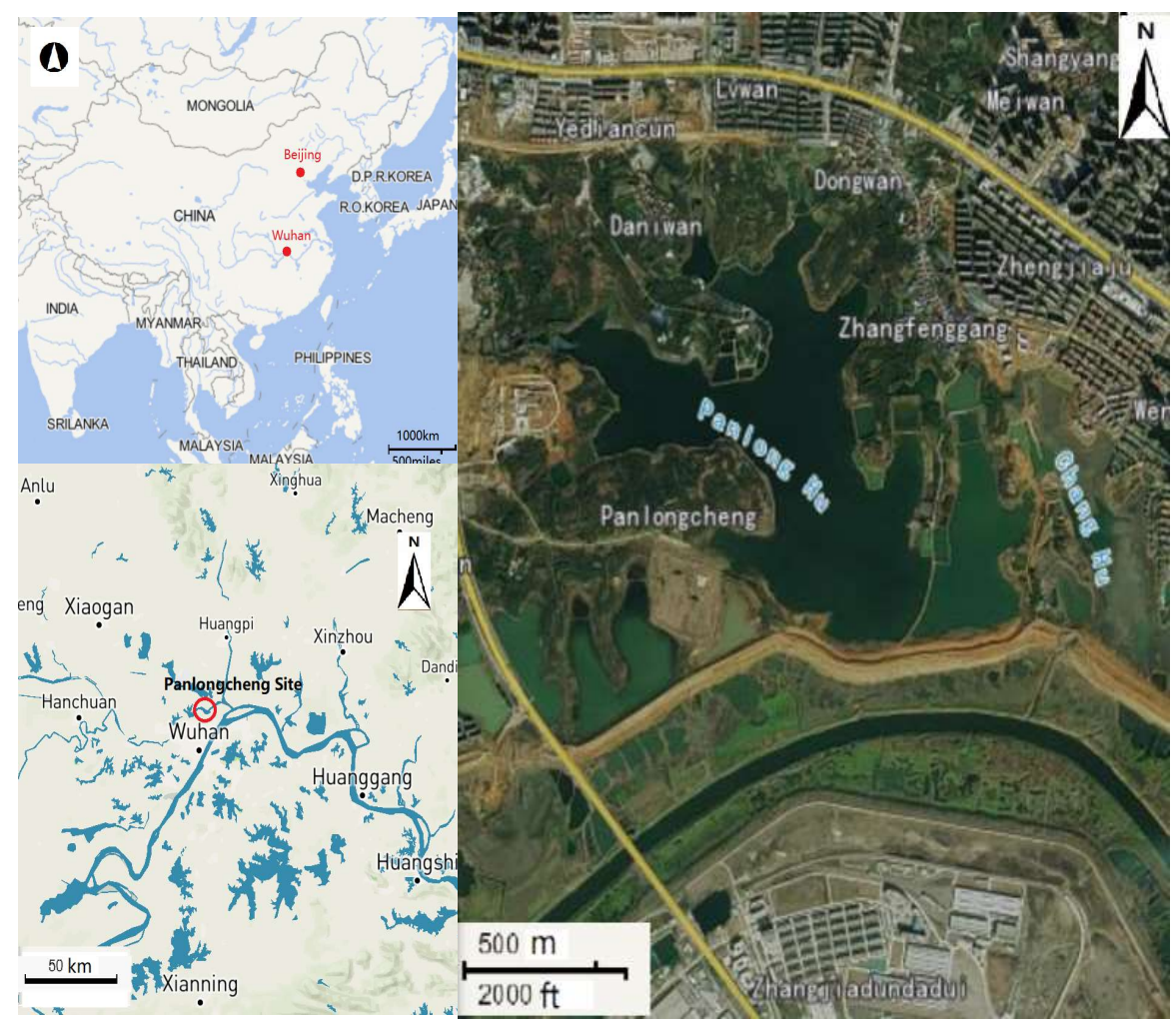

Figure 1. Research area. This figure shows the location of Wuhan in China, the location of the Panlongcheng site in Wuhan and the satellite image of the Panlongcheng site.

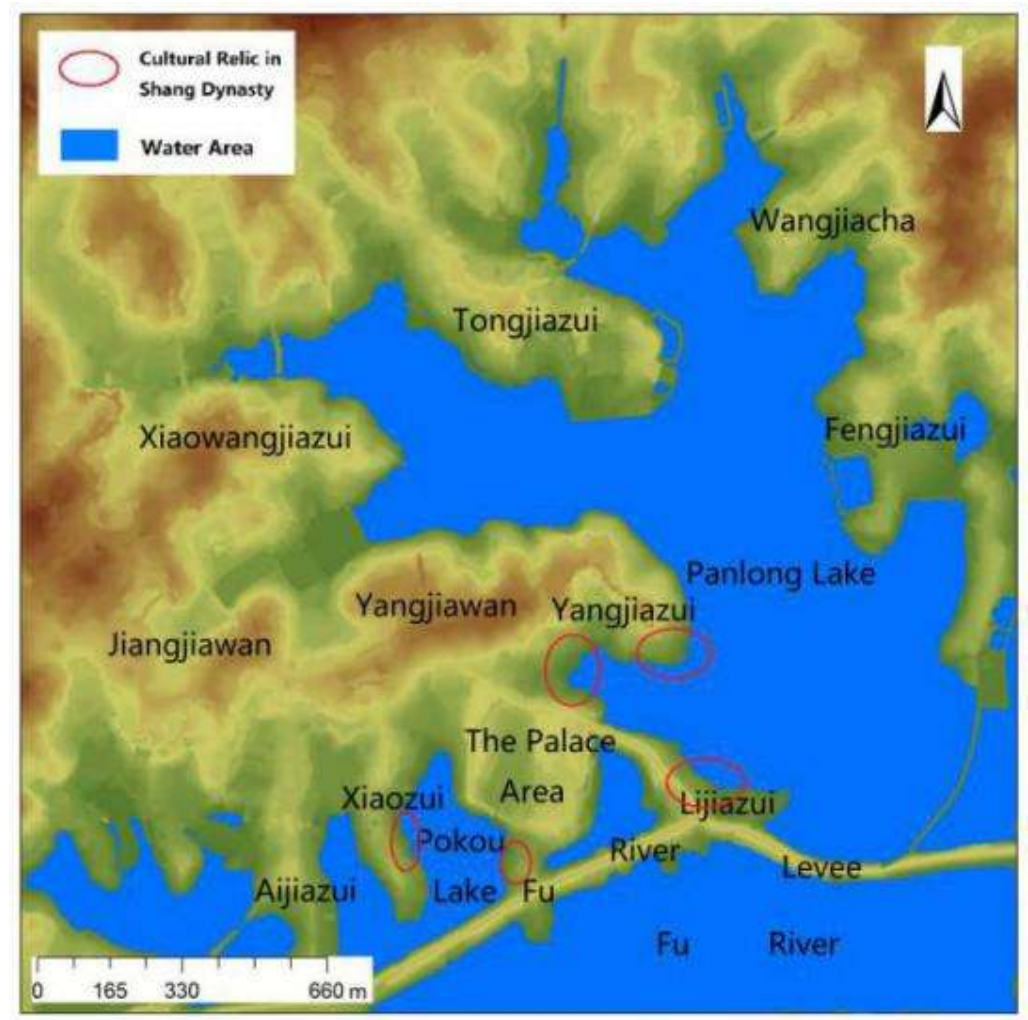

Figure 2. Distribution of cultural sites in the Panlongcheng Site. This figure shows the geographical environment and distribution of cultural relics of the Panlongcheng site. The cultural sites of Shang Dynasty are widely distributed in the areas marked by the red circle in the picture. 


\subsubsection{Materials}

Digital line drawing (DLG) is a geographic information vector data set that expresses topographic features in the form of points, lines, areas or map-specific graphic symbols. The digital mapping method was used to generate the DLG file of the Panlongcheng site. In 2018, UAV aerial photography was conducted in the Panlongcheng area, and then the digital photogrammetry software vintuozo system was used to carry out stereoscopic mapping of aerial photos to obtain the DLG Data of the study area. We use ArcGIS 10.2(Redlands, CA) to edit the obtained data and finally produce the result data. The specific method is as follows: firstly, the aerial photography is used to judge and draw the elements of water system, traffic and artificial features. Then, according to the results of aerial photo judgment, the elements of the drawing shall be comprehensively checked in the field. Finally, the elements are collected and edited according to the results of field verification [20]. In the production of 1:2000 scale digital line drawing of the Panlongcheng site, the contour line, road water system and some artificial facilities (such as large houses and dams) of the site are retained for further operation. As shown in Figure 3, the digital line graph of the Panlongcheng site is used to restore the landscape.

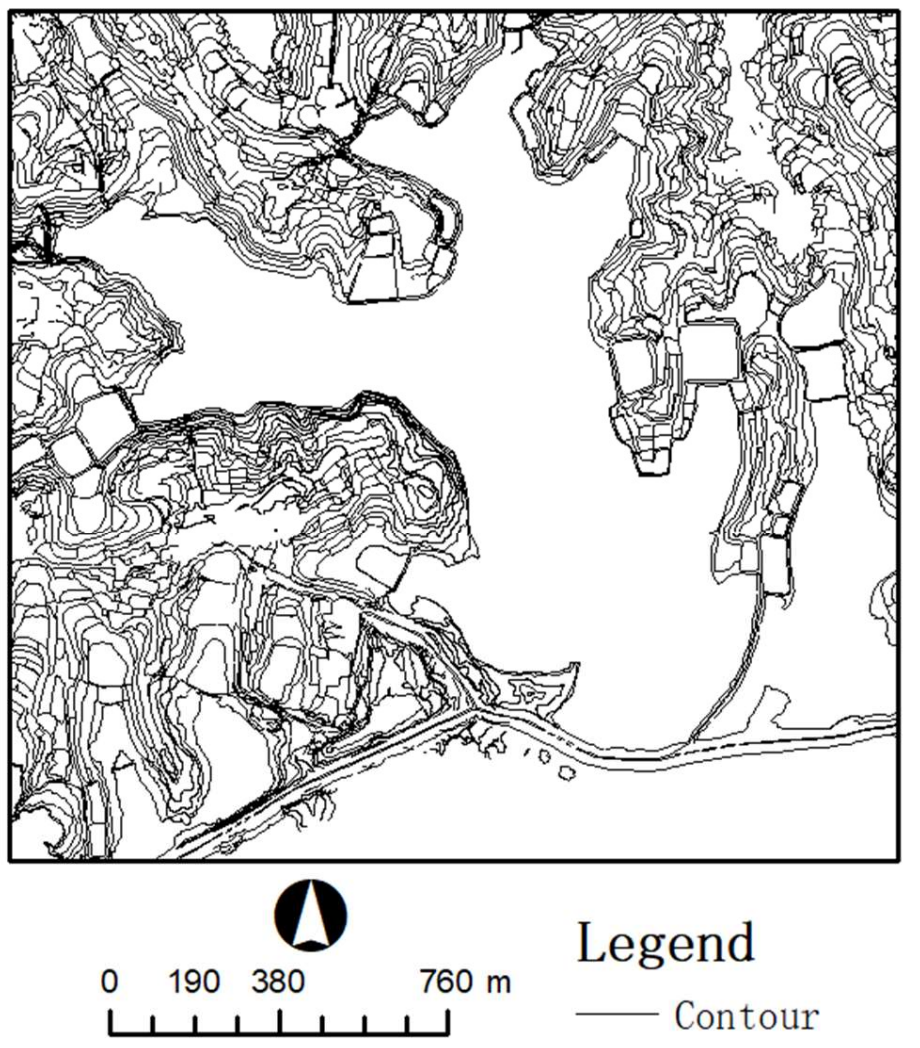

Figure 3. Digital line drawing (DLG) of the Panlongcheng site in modern time.

From 2012 to 2016, systematic archaeological exploration was carried out in the land area of the Panlongcheng site. As shown in Figure 4,the strata of the Panlongcheng site area can be generally divided into three layers from top to bottom: the first layer is the topsoil, which is accumulated by modern human activities; the second layer is the cultural layer of the Shang Dynasty, including ash pits, building foundation sites, tombs and other cultural deposits formed by human activities in the Shang Dynasty; the third layer is raw soil, which does not contain any human activity traces, belonging to the natural accumulation formed before the appearance of the Panlongcheng site. Relevantly, we can divide the landscape restoration results of the Panlongcheng site into three different periods: before the Shang Dynasty, after the Shang Dynasty and modern time. 


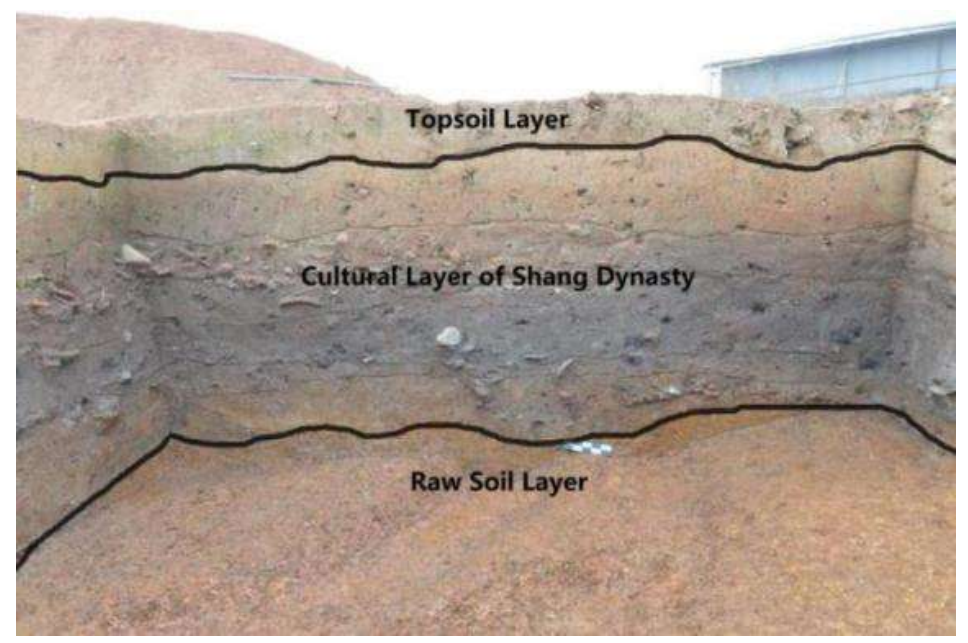

Figure 4. Stratum of the Panlongcheng Site in land area. From the surface to the underground, there are topsoil layer, Shang Dynasty cultural layer and raw soil layer, which represent the accumulation of human activities in modern times, Shang Dynasty and before the Shang Dynasty.

In this exploration, about 15,000 boreholes are arranged along the north-south direction with a spacing of $10 \mathrm{~m}$, as shown in Figure 5a [6]. Taking Yangjiawan as an example, there are two kinds of borehole in the exploration. The stratum of first kind is divided into topsoil layers, cultural layers and raw soil layers in the areas where people in the Shang Dynasty lived. For the second kind, there were only topsoil layers and raw soil layers in the area where no one lived during the Shang Dynasty. The territory of drilling data of is similar to the excavation area. During archaeological excavation, archaeological drilling holes are carried out in the area shown in Figure 5a. As shown in Figure 5b, drilling holes were set every $10 \mathrm{~m}$ in the east-west or north-south direction of the area. Each drilling hole takes soil about $2 \mathrm{~m}$ below the ground to ensure that each hole penetrates the topsoil and cultural layer and touches the raw soil layer. During archaeological exploration, we try to cover the target area with exploratory holes. However, due to the existence of ground buildings or complex environment, which is not suitable for exploration, a small number of areas are not covered by drilling holes.

Topographic mapping and underwater archaeological exploration of Panlong lake by using single beam ultrasonic sounder was carried out in 2016. Surveying and mapping the underwater topography of Panlong lake is the basis for exploring underwater archaeological relics. Panlong lake covers an area of about 1 square kilometer and the water depth is about $0-4 \mathrm{~m}$. For the surveying and mapping of the lake topography, we use the joint operation of the survey ship equipped with RTK Positioning System and single beam sounder, and select the draft of $0.9 \mathrm{~m}$, A small fishing boat with a length of about $11 \mathrm{~m}$ was equipped with zhonghaida H32 RTK and zhonghaida hd-max single beam bathymeter to survey the underwater terrain of Panlong lake. The sounding accuracy of hd-max sounder is $\pm 1 \mathrm{~cm}$ and the sounding range is $0.15-300 \mathrm{~m}$. Considering the draft of the measuring ship, the mapping work is not carried out for the water area with a water depth of less than $1 \mathrm{~m}$. Then, we drilled underwater in Panlong lake. The drilling equipment is a professional small-scale detachable water drilling platform, which is composed of a rubber buoy, carbon fiber platform, aluminum alloy drilling frame and drilling tools, and a small kayak is used for cooperation. On the built drilling platform, we drilled and sampled holes parallel to the lake bank with a spacing of $50 \mathrm{~m}$, densified them in the Yangjiawan area where cultural relics were found, observed the stratigraphic distribution in the exploratory holes on site and registered archaeological information. Due to the different depths of the lake bottom and the different thickness of cultural accumulation, some exploratory holes are more than $10 \mathrm{~m}$ deep, which makes it difficult to operate on water. We have drilled three exploration zones in Panlong lake, and the number of exploratory holes is more than 100. In this paper, 
the underwater survey data obtained from the surveying and mapping exploration are used for research [19]. The distribution of underwater survey points is shown in Figure 6.

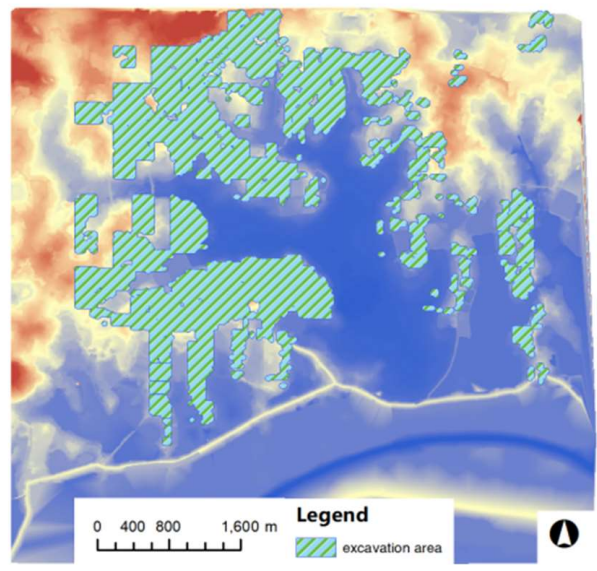

(a)

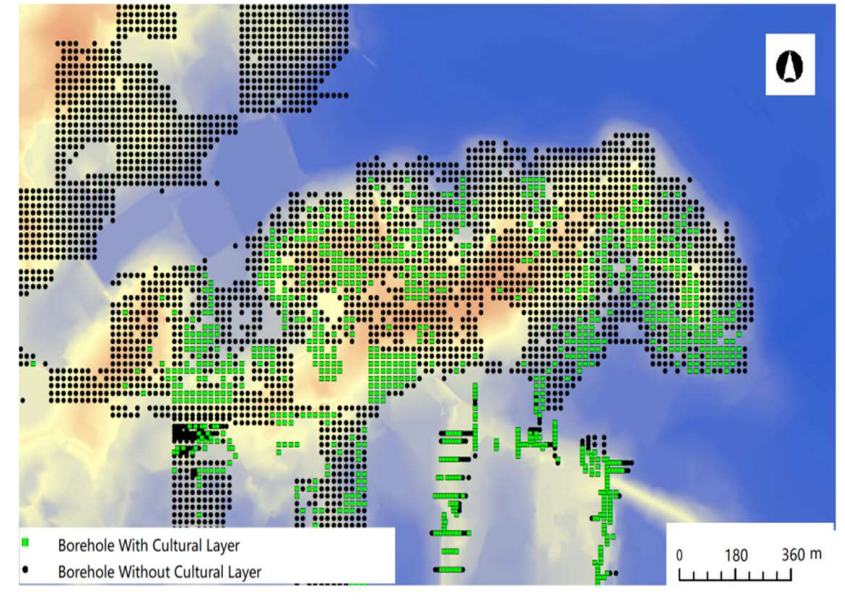

(b)

Figure 5. (a) Excavation area of archaeological boreholes in the Panlongcheng site; (b) Distribution of boreholes. The area covered by green exploratory holes represents the area with Shang Dynasty cultural layer, and the area covered by black exploratory holes represents the area without Shang Dynasty cultural layer.

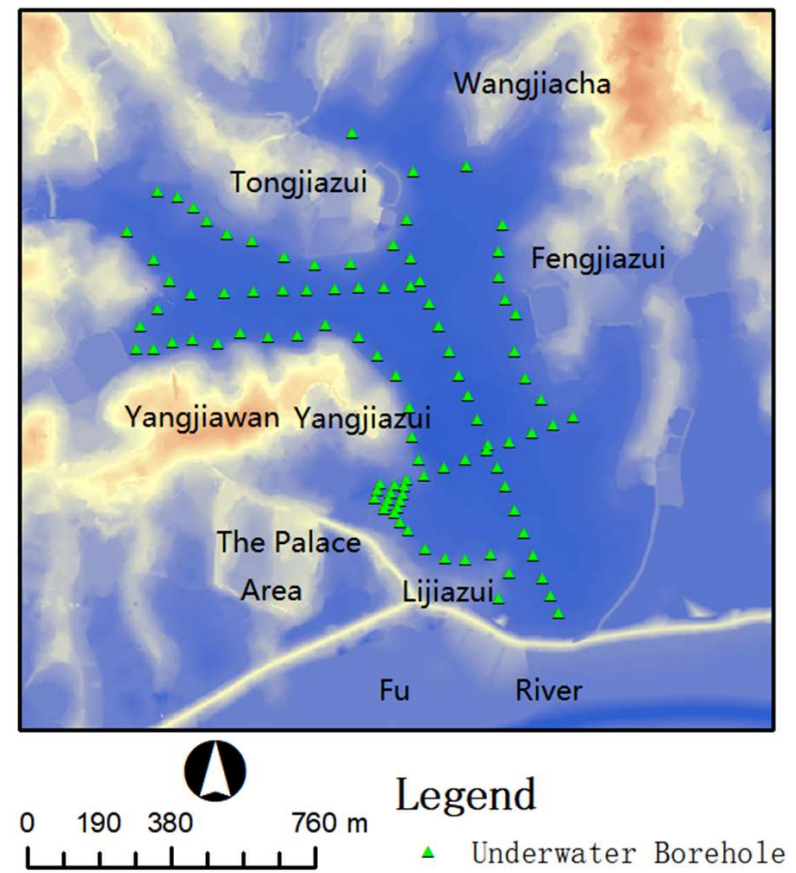

Figure 6. Distribution of underwater boreholes by single beam sounder of the Panlongcheng Site.

The single beam sounder transmits short-pulse sound waves vertically by transducer. When the pulse sound wave meets the riverbed, the reflected echo will return to sonar and be received by the transducer. Single beam depth sounder adopts single point along the measurement track. Therefore, there is no information collected between points. The data obtained from the bottom of the lake are discontinuous. Therefore, interpolation is necessary to reconstruct the underwater terrain of Panlong lake. The stratum of the soil layer at the bottom of the lake is similar to that in the land area. Topsoil layer is replaced by silt layer and raw soil layer is replaced by hard clay layer. According to the results of 
underwater measurement, the bottom of Panlong lake is relatively flat, with silt layer of about $2 \mathrm{~m}$ height. The remains of the Shang culture were found below the water surface near the west bank of Panlong lake, widely distributed in the lake area between Yangjiazui and Lijiazui. The underwater boreholes of Panlong lake are widely distributed [21].

Combined with topography in the land area, the landscapes of the Panlongcheng site in the Shang Dyansty and before the Shang Dynasty are obtained by removing the topsoil layer, silt layer, cultural layer and artificial construction.

\subsection{Methodology}

To before the Shang Dynasty, after the Shang Dynasty and modern time and analyze the land-water distribution in different periods, the specific process is as follows. Firstly, to obtain the DEM base map without artificial features, the raster data and digital line map are edited to remove the artificial features of modern time and Shang Dynasty. Then, the TIN (TIN, Triangulated Irregular Network) and DEM (DEM, Digital elevation model) base map is generated. The Delaunay triangulation algorithm is used to generate TIN. Bilinear interpolation is used to fit the DEM from corresponding TIN. Secondly, the soil layer to be stripped is fitted for further steps. The data obtained from archaeological exploration in the land area and underwater area were combined to generate the DEM of soil layers to be stripped. According to the archaeological exploration data, we can generally divide the strata of the Panlongcheng site into topsoil combined with silt layer, the Shang culture layer and raw soil combined with hard clay layer from top to bottom. To obtain the landscape of the Panlongcheng site in the Shang Dynasty, it is necessary to remove modern artificial features, and then peel off the topsoil combined with silt layer. When restoring the landscape of the site before Shang Dynasty, it is necessary to remove the all the artificial features, and then peel off the topsoil combined with silt layer and the Shang culture layer. Finally, the topographical changes in the Panlongcheng site are analyzed and visualized with historical water level changes. The abstract is shown in Figure 7.

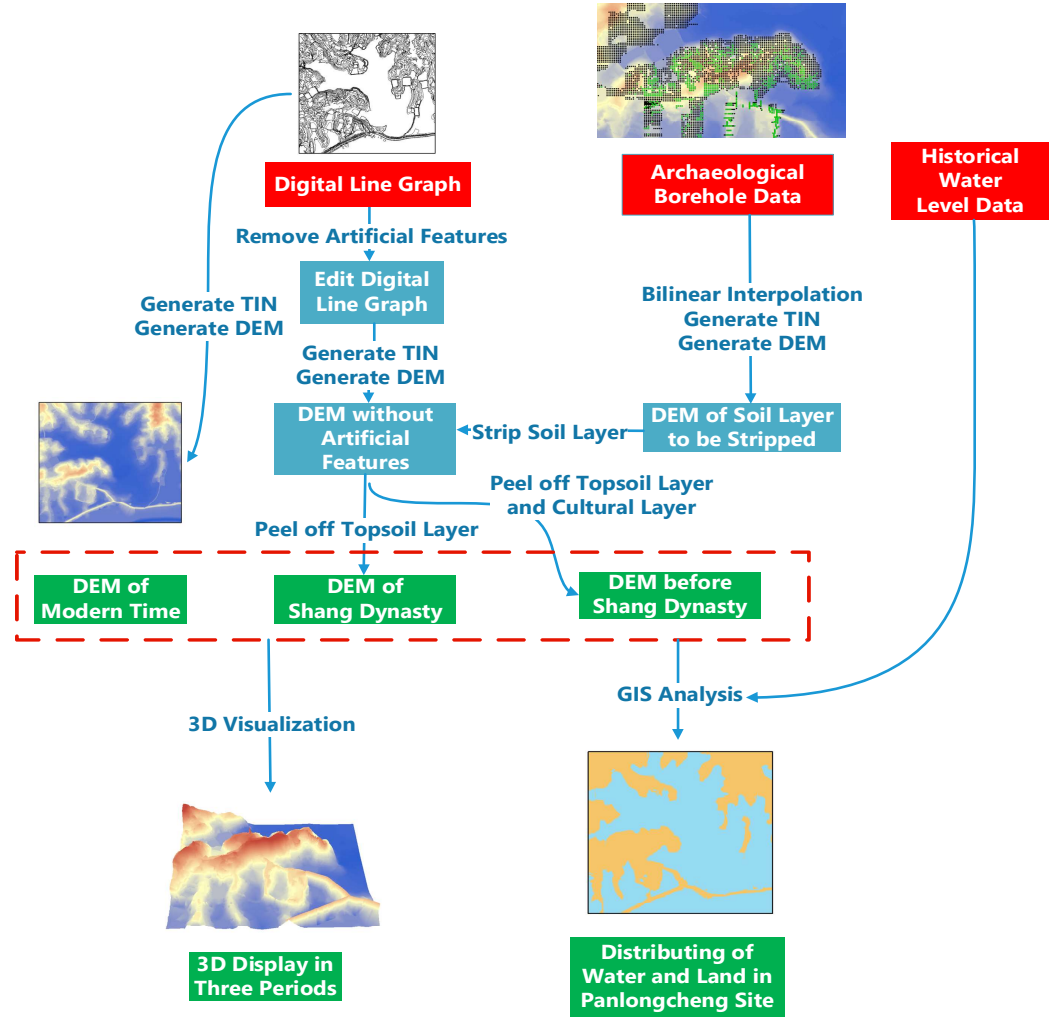

Figure 7. Method flow chart of restoring landscapes and analyzing water-land distribution of the site. 


\subsubsection{Obtain DEM Base Map with Artificial Features Removed}

In this study, to obtain the DEM before and after the Shang Dynasty, the digital line graph and raster data of the Panlongcheng site were edited, and the contemporary artificial features and the artificial buildings of the Shang Dynasty were removed. The specific process is as follows: firstly, the data were edited to obtain the base map before and after the Shang Dynasty in which the artificial features are removed. ArcGIS(Redlands, CA) was used to edit the digital line graph of the Panlongcheng site. We deleted or corrected the abnormal elevation value, removed the houses, dams and other modern artificial facilities, combined the results with the elevation points to generate TIN, and used ArcGIS to carry out bilinear interpolation methods to generate a DEM base map with modern artificial features removed. Then, the TIN and DEM with the artificial features removed from the Shang Dynasty were obtained by using the same method. As shown in Figure 8, edited DLG without modern artificial features and DLG without all artificial features are shown.

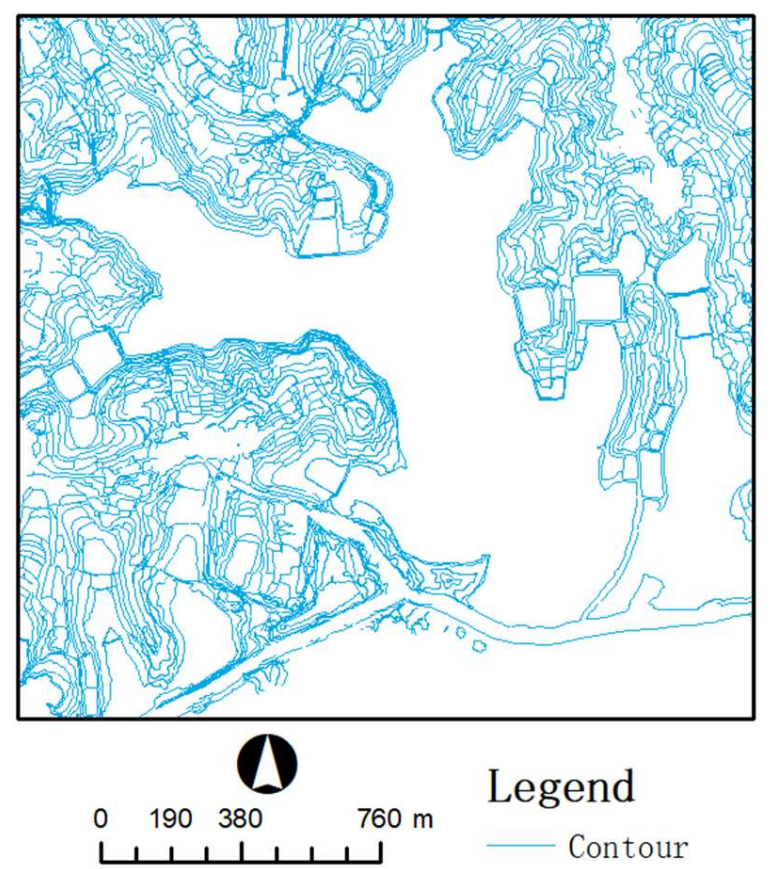

(a)

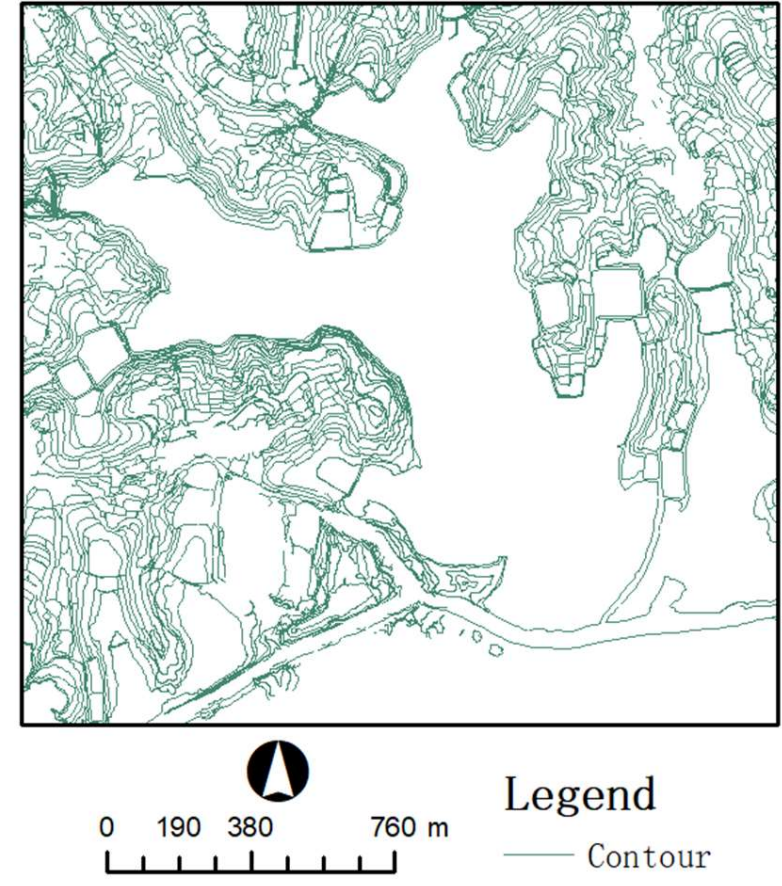

(b)

Figure 8. DLG before and after Shang Dynasty with artificial features removed: (a) DLG without modern artificial features; (b) DLG without all artificial features.

With ArcGIS 10.2(Redlands, CA), the results of edited contour lines from 8 (a) and 7 (b) are combined with elevation points to generate TIN, as shown in Figure 9a,b. The TIN model uses a series of connected triangles to fit the surface. It is often used to construct digital terrain models, especially DEM. TIN has many advantages in reflecting complex surface. It has been widely used in digital system, modeling and analysis of ground surface. The most commonly used method to generate TIN is a Delaunay subdivision algorithm. The Delaunay triangulation network is a set of adjacent and non-overlapping triangles. The circumcircle of each triangle does not contain other points [22]. Taking the rampart as an example, it can be seen that the topography of the Panlongcheng site was obviously different due to human activities before and after the Shang Dynasty.

The method of bilinear interpolation is used to generate the DEM of removing modern artificial features and DEM without all artificial features from corresponding TIN, as shown in Figure 10a,b. The core idea of bilinear interpolation is linear interpolation in two directions. In mathematics, bilinear interpolation is an extension of linear interpolation 
for interpolating functions of two variables (e.g., $\mathrm{x}$ and $\mathrm{y}$ ) on a rectilinear 2D grid. The key idea is to realize linear interpolation in one direction firstly, and then in the other direction. Although each step is a linear interpolation in the resampling values, the interpolation as a whole is not linear but rather quadratic in the resampling values [23]. In short, input and output data of obtaining DEM base map with artificial features removed are the DLG (contour line representing height) and DEM base map. In this step, bilinear interpolation is realized in the ArcGIS software.

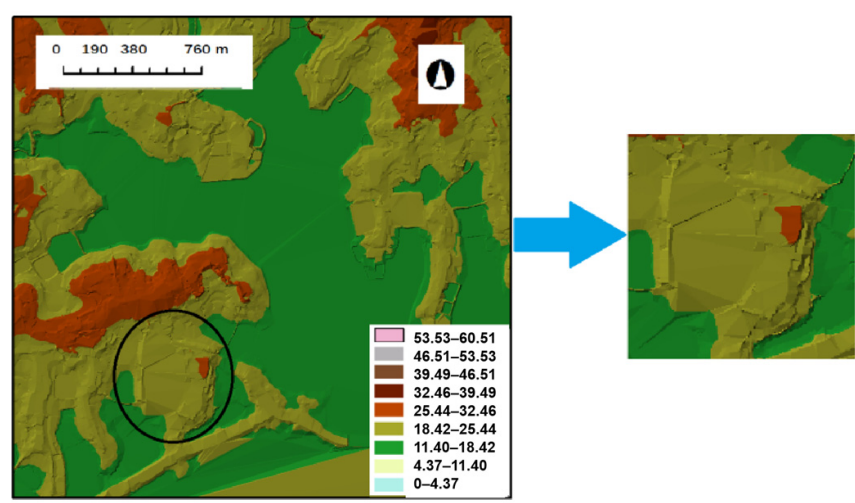

(a)

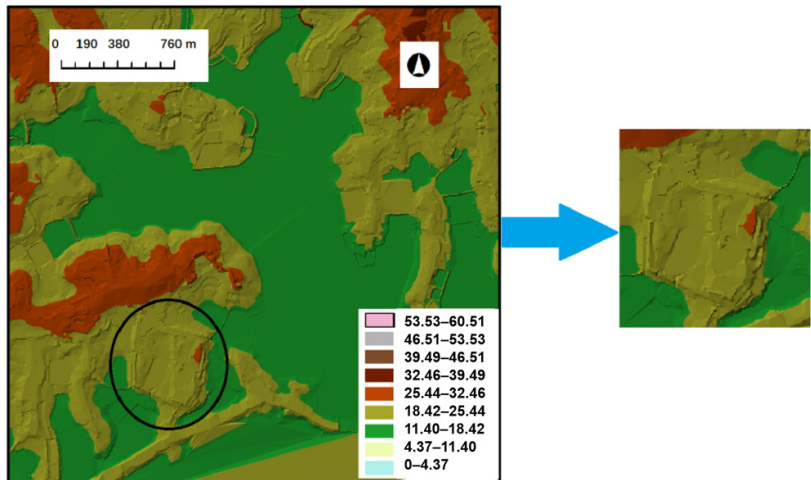

(b)

Figure 9. Triangulated Irregular Network(TIN) with artificial features removed; the palace area is showed separately: (a) TIN without modern artificial features; (b) TIN without all artificial features.

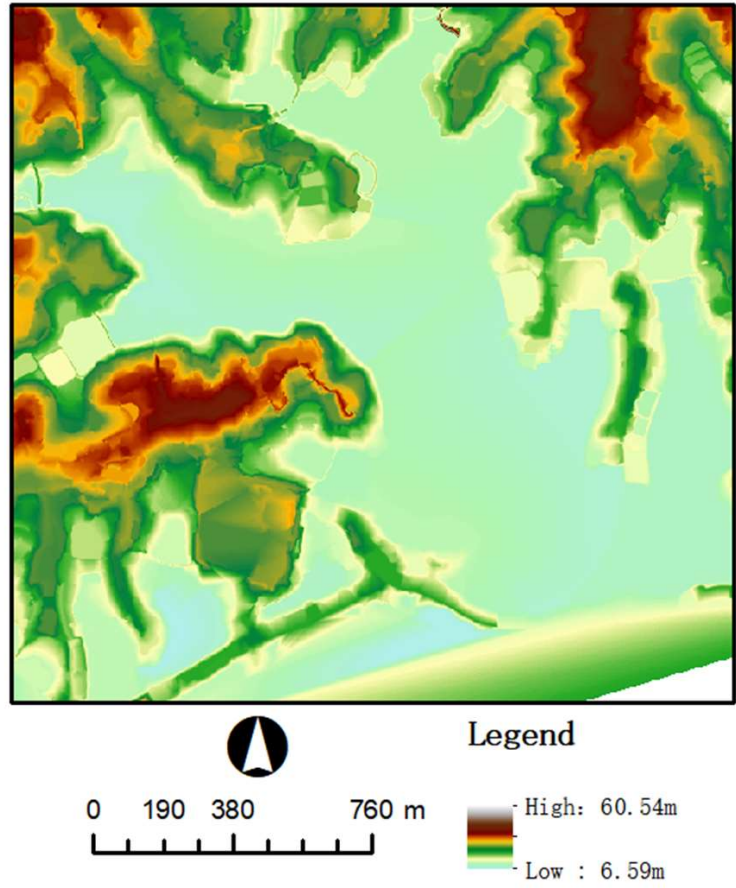

(a)

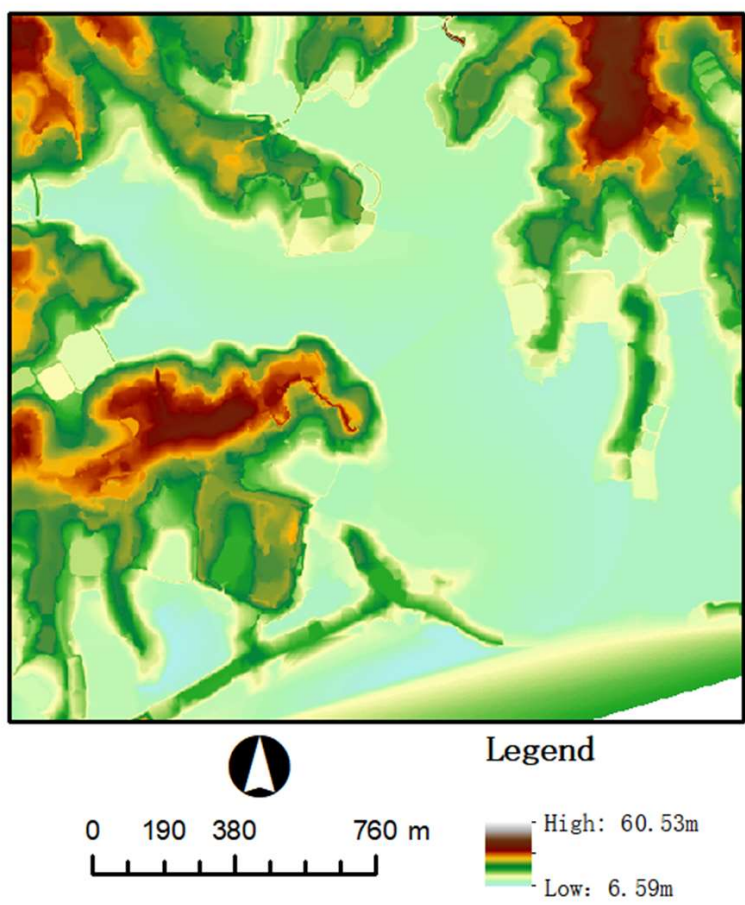

(b)

Figure 10. Digital Elevation Model(DEM) with artificial features removed: (a) DEM without modern artificial features; (b) DEM without all artificial features.

\subsubsection{Fit Soil Layers to Be Stripped and Restore the Terrain and Landscape}

In order to restore the terrain in the Shang Dynasty, it is necessary peel off the topsoil layer and silt layer from DEM base map with modern artificial features removed. Similarly, 
when restoring the terrain of the site before the Shang Dynasty, it is necessary to peel off the topsoil layer, silt layer and the cultural layer from the DEM with modern and Shang artificial features removed. Therefore, it is very important to fit the surface layer needed to be stripped.

The TIN of the soil layer to be stripped is generated from the thickness of the topsoil layer, silt layer and the cultural layer, and the DEM is obtained by using the bilinear interpolation. When we fit soil layers to be stripped, input and output data are points representing drilling holes and DEM to be stripped. In this step, we generalize DEM by bilinear interpolation in ArcGIS(Redlands, CA) software. As shown in Figure 11, it is necessary to strip up to $3 \mathrm{~m}$ of topsoil and silt soil layer on the basis of the results of DEM obtained in the previous step to restore the landscape in the Shang Dynasty. Moreover, to restore the landscape before the Shang Dynasty, up to $4.75 \mathrm{~m}$ of topsoil and silt accumulation and cultural layers should be stripped too. In addition, the depth of sludge accumulation in Panlong lake is thicker than that in the land area. In order to obtain the DEM before the Shang Dynasty and during the Shang Dynasty, the DEM of stripped stratum is subtracted from the corresponding DEM obtained in Section 3.1.

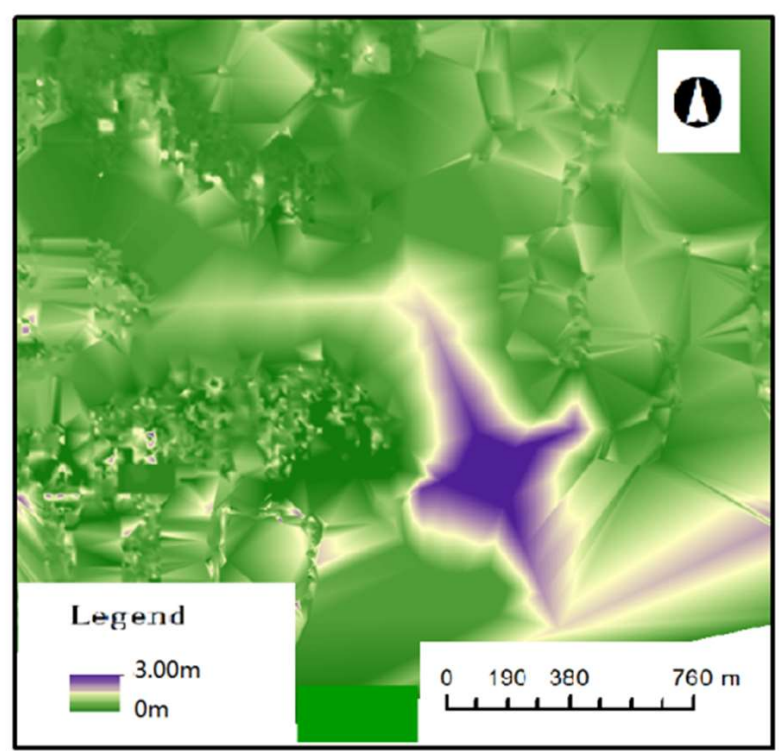

(a)

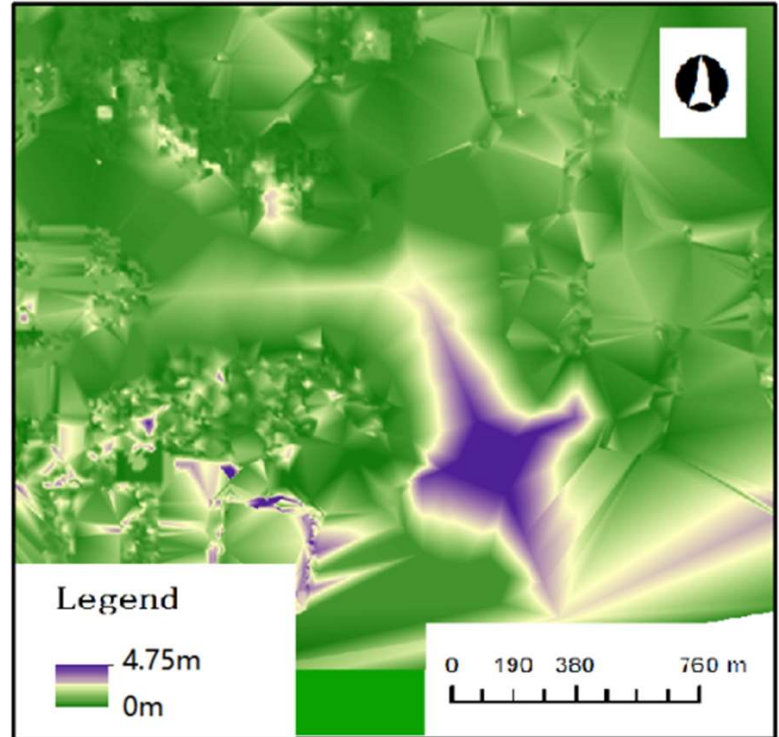

(b)

Figure 11. DEM of soil layer to be stripped: (a) topsoil layer to be stripped; (b) topsoil and cultural layer to be stripped.

\subsubsection{Analyze the Water-Land Distribution in Different Periods with the Historical Water Level}

Nowadays, Panlong lake and Pokou lake have been turned into artificial fisheries, and the water level is kept between $19.5 \mathrm{~m}$ and $22.6 \mathrm{~m}$ throughout the year. The Fu River levee lies between the Fu River, Panlong lake and Pokou lake. The three waters are independent and disconnected from each other. When the rainy season comes in summer and autumn, the area of rivers and lakes around the Panlongcheng site accounts for about $50 \%$ of the total area of the site. Affected by the barrier of lake, the whole landform of the site is broken. The Palace area, the core of the Panlongcheng site, is also surrounded by the lake on the east, south and west sides. When the flood season comes, the rising lake water can even submerge the Palace area. Because of the serious threat of flood disaster, the landscape settlement with a large water area is obviously not suitable for human survival, which is also different from the land and water spatial pattern of most cities in the Shang period.

However, archaeological evidence shows that the water level of rivers and lakes in the Panlongcheng site of Shang Dynasty was at least $5.1 \mathrm{~m}$ lower than the contemporary 
water level. Under such low water level conditions, the water area of rivers and lakes in the Panlongcheng site would be greatly reduced, which accounted for about $15 \%$ of the total area of the site. Therefore, there was vast land space for the construction of ramparts and the development of the whole city. The outer side of Panlongcheng ramparts is no longer besieged by water. Instead, it is connected with several hillocks around the ramparts, such as Lijiazui, Wangjiazui, Xiaozui and Yangjiawan. Under these circumstances, it is convenient for interacting and communicating between the Palace area and the surrounding functional areas (burial area, manual workshop area, building residential area, etc.). It can be seen that the low water level around the Panlongcheng site in the Shang dynasty established an important natural and geographical foundation for the rise of Panlongcheng culture. However, due to the rising water level of rivers and lakes, the landscape with rivers and lakes crisscrossed and broken land area is formed.

With ArcGIS 10.2(Redlands, CA), the restored landscape and the water level of modern and the Shang Dynasty in different seasons is used to analyze the water-land distribution of the Panlongcheng site in different periods. Taking $22.6 \mathrm{~m}, 21.1 \mathrm{~m}$ and $19.5 \mathrm{~m}$ as water levels in the rainy season, normal season and dry season, water-land distribution in the present day is analyzed. Similarly, water-land distribution in the Shang Dynasty was displayed with ancient terrain and water levels at $17.5 \mathrm{~m}, 16 \mathrm{~m}$ and $14.4 \mathrm{~m}$.

\section{Results and Discussion}

\subsection{Results of Terrain and Landscape Restoration of the Panlongcheng Site}

DEM in three periods is shown in Figure 12. We can divide the process of the regional geomorphic changes into three stages: before the Shang Dynasty, in the Shang Dynasty and modern times. Due to the environment changes and human activities, there are significant differences in landscapes in different stages. Furthermore, the overall layout of the Panlongcheng site should be considered with the water level change in the early Shang Dynasty. In Yangjiawan, Wangjiazui, Lijiazui and Xiaozui areas where palace buildings and tombs of the Shang Dynasty are clustered, although the elevation in the Shang Dynasty was slightly lower than that in the modern time, the water level of Panlong Lake in the Shang Dynasty was lower than that in the contemporary period. Due to the rising water level of Panlong lake, some sites may be merged and eroded by Panlong lake nowadays.

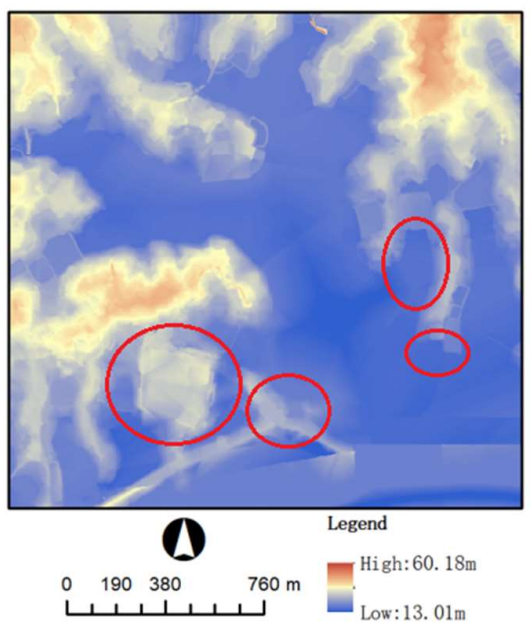

(a)

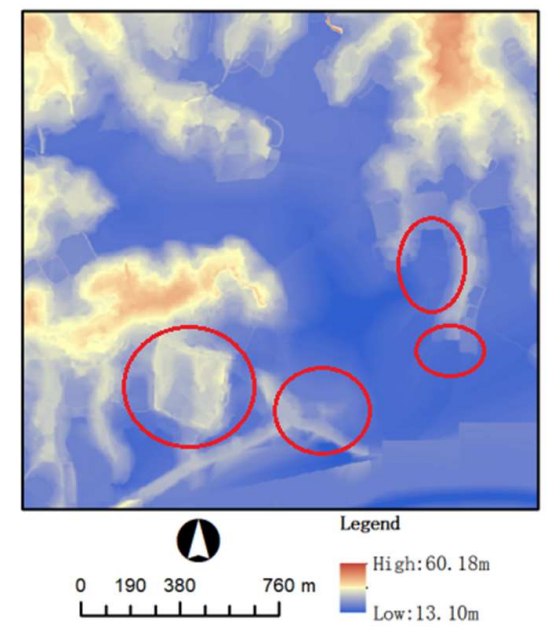

(b)

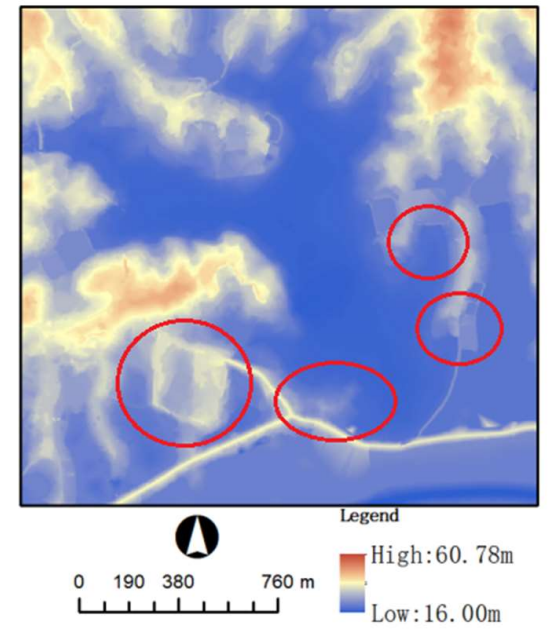

(c)

Figure 12. DEM of the Panlongcheng site in different periods, areas with obvious geomorphic changes are marked by red circles: (a) before the Shang Dynasty; (b) in the Shang Dynasty; (c) in the modern time.

With ArcGIS 10.2(Esri, Redlands, CA, USA), the landscapes of three different periods are displayed in three dimensions. Taking ramparts in the Palace area of Shang Dynasty 
as an example, the results is shown in Figure 13. The Palace area is built with the east and west ridges near the Panlongcheng site. The ramparts was built in the south, which became a square-shaped earthen wall. Compared with Figure 13a,b, it can be seen that the southern ramparts was built during the Shang Dynasty. Compared with 13 (c), the construction of Fu River levee can be displayed. In addition, for the area between Palace area and Yangjiazui, there has been an obvious topographic evolution with the passage of time, which is caused by the change in water level and erosion.

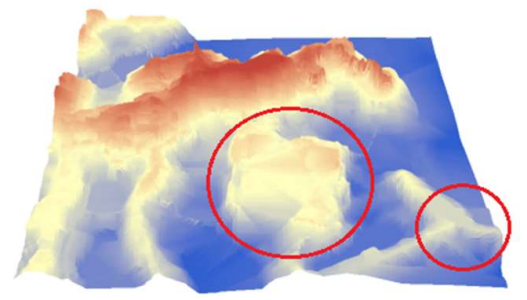

(a)

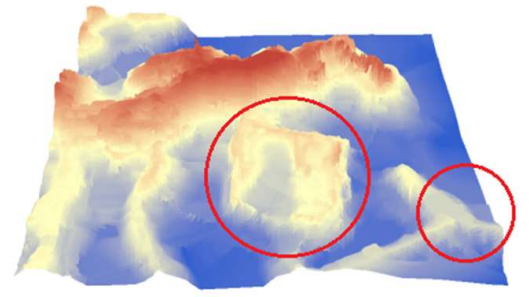

(b)

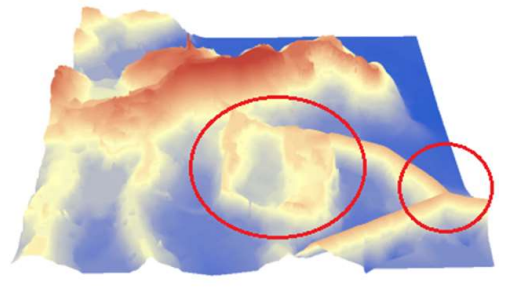

(c)

Figure 13. Visualization of landscapes of the Panlongcheng site in different periods: (a) before the Shang Dynasty; (b) in the Shang Dynasty; (c) in modern times.

As shown in Figure 13a, before the Shang Dynasty, the Panlongcheng site was a natural hillock on the North Bank of the Fu River, a tributary of the Yangtze River. All the narrow and long hillock fields naturally extended. Between the hills the low-lying flat land appeared. There was no artificial facilities in the area.

In the Shang Dynasty, the population of the Panlongcheng site increased significantly. In about 100 years, it developed rapidly from rare natives to the largest city in the middle reaches of the Yangtze River and became the central city of the region. In the Palace area, a rammed earth city wall with a length of $290 \mathrm{~m}$ from north to south and $260 \mathrm{~m}$ wide from east to west was built by the local people taking soil from the surrounding area. The two south-north-oriented hills became the east and west ramparts of the Panlongcheng site, and the depressions between the two natural hills was also rammed into the south and north walls. At the same time, the depression around the ramparts was transformed into a moat. Local residents in the Shang Dynasty also built a rammed platform in the northeast of the ramparts, and three large-scale palace buildings (F1, F2, F3) were constructed on the platform. As shown in Figure 14, among them, the F1 palace building is the largest one, $39.8 \mathrm{~m}$ in length from east to west and $12.3 \mathrm{~m}$ wide from north to south. It is the largest palace building of the Shang Dynasty found in the Yangtze River basin so far [24].

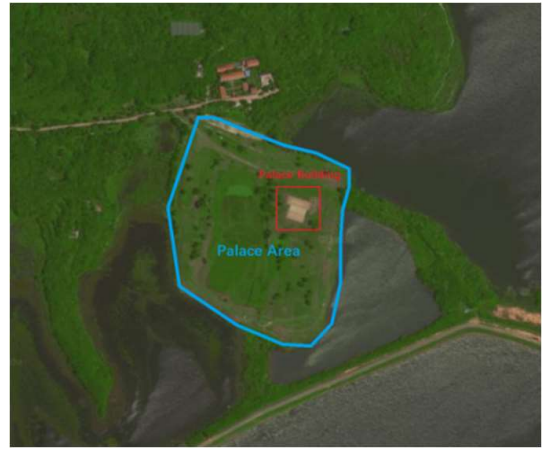

(a)

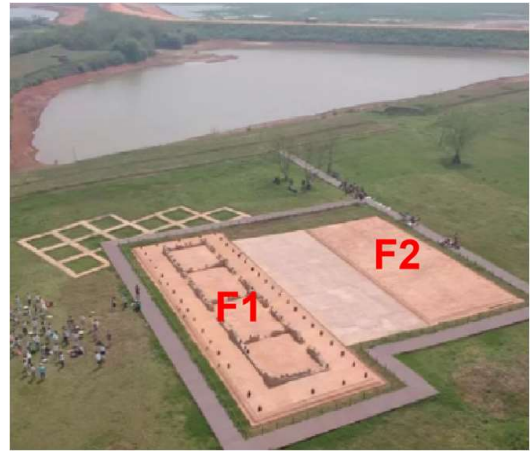

(b)

Figure 14. Distribution of palace area and location of palace buildings: (a) satellite image map of palace area; (b) distribution of palace buildings in palace area, F1 and F2 are the sites of the palaces of the Shang Dynasty. 
The Panlongcheng site was abandoned after the Shang Dynasty. Archaeological investigation shows that extremely few people lived in this area during thousands of years after the Shang Dynasty. After the founding of the People's Republic of China, the Panlongcheng site, located in the lower reaches of the Fu River, was seriously flooded. In order to control the flood disaster, the local government organized villagers to build the Fu River levee on the North Bank of the Fu River. After the construction of the dike, the Panlong lake was no longer connected with the Fu River. Water gathered in rainy season is difficult to leak out, which makes Panlong Lake at a high water level all year round. In the rainy season, due to the barrier of artificial dikes on both sides of the river, the water level of Fu River rises sharply. Therefore, the water level of rivers and lakes in the Panlongcheng site rose significantly, and the lakeside area of the Panlongcheng site was submerged.

\subsection{Land and Water Distribution in Different Periods}

As shown in Figure 15, water-land distribution in the Panlongcheng site in different water level in modern time and the Shang Dynasty is displayed. For the modern Panlongcheng site, the water-land distribution in the rainy season is significantly different from that in the dry season. In the rainy season, both the water area of Fu River and Panlong lake were significantly widened. At present, the area of the Panlongcheng site is about $3.5 \mathrm{~km}^{2}$. When rainy season comes, the water area will exceed $2 / 3$ of the total site area. Such a vast water area will divide the site into several peninsula-shaped hillocks.

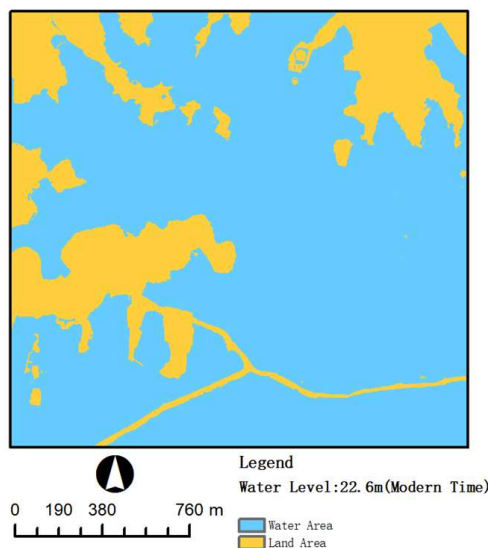

(a)

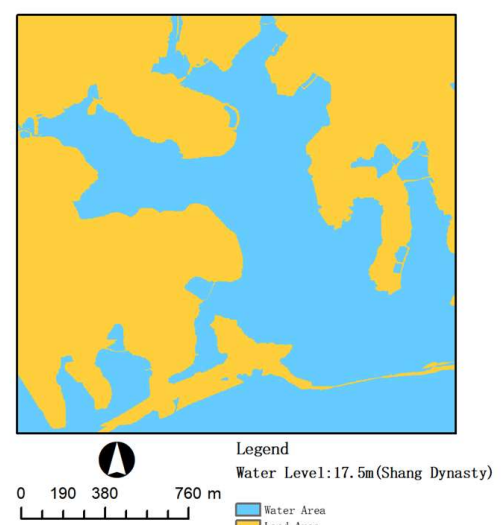

(d)

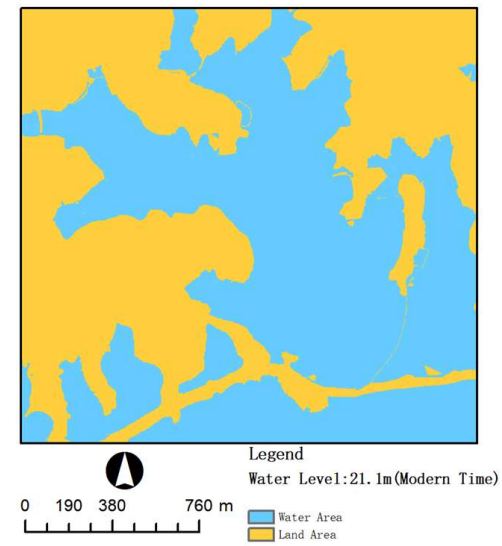

(b)

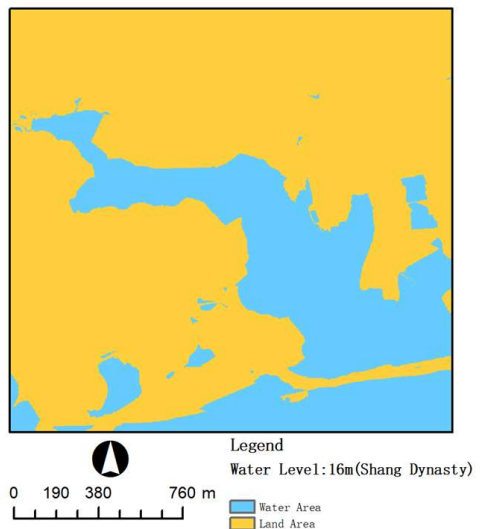

(e)

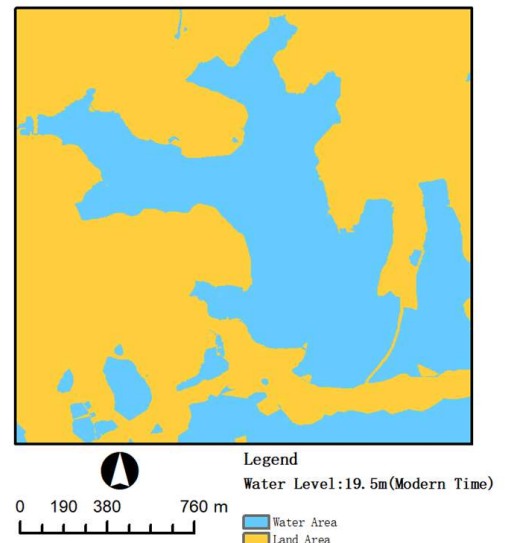

(c)

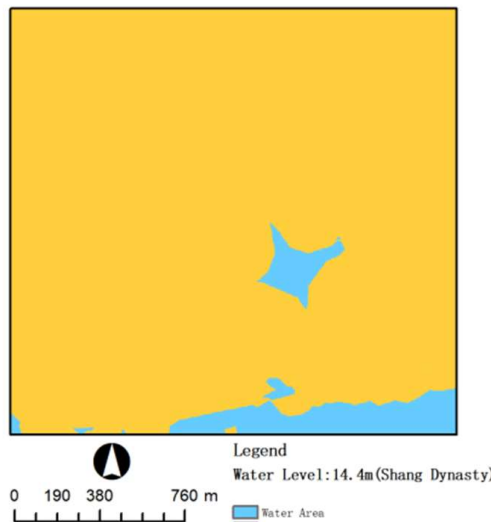

(f)

Figure 15. Water-land distribution in the panlongcheng site in different water levels in modern times and in the Shang Dynasty: (a) rainy season in modern time; (b) normal season in modern times; (c) drought season in modern times; (d) rainy season in the Shang Dynasty; (e) normal season in the Shang Dynasty; (f) drought season in the Shang Dynasty. 
In terms of the modern geomorphic features of the Panlongcheng area, the terrain is low and flat, and the low hills crisscross with lake branches and river channels. During the rainy season, the rising flood in the lower reaches of the Fu River will submerge part of the Panlongcheng site, and the flood in the main stream of the Yangtze River will also pour into the Fu River, aggravating the flood disaster in Panlongcheng area. Therefore, the Panlongcheng site has been under serious flood threat for a long time in the past 100 years. At the same time, the region is also a high incidence area of schistosomiasis. The annual flooding period coincides with the outbreak period of schistosomiasis [25]. These factors make the Panlongcheng area populated sparsely and developed slowly. After the founding of the People's Republic of China, a large number of artificial dikes and dams were built in this area, and the disasters of flood and schistosomiasis were greatly solved.

In such an uninhabitable area, appearance of a large-scale city in the early Shang Dynasty is hard to understand in terms of modern geographical environment. When the water level of the river and the lake drops to $17.5 \mathrm{~m}$, the landform of the Panlongcheng site is bound to change significantly. Comparing with landscape in the Shang Dyansty, except the difference of dike and other infrastructure, the water level of Panlong Lake was lower and the water area was small in the Shang Dynasty. There were large areas of land between Yangjiazui, Yangjiawan and Palace area. In the survey of underwater archaeological drilling holes, a large number of remains from the Shang Dynasty culture layer have been found in this area.

It can be seen that the water level of rivers and lakes near the Panlongcheng site in the early Shang Dynasty was significantly lower than that in modern times. In the Shang Dynasty, when the highest water level of Panlong lake and Fu River was about $17.5 \mathrm{~m}$, Panlong lake was only a small water area with an area of no more than $0.4 \mathrm{~km}^{2}$, and the width of Fu River channel was less than $100 \mathrm{~m}$. Seasonal fluctuation of river and lake water level considered, the water level of Panlong lake and Fu is lower in the normal season, and Panlong lake would be completely dried up in the dry season in the Shang Dynasty. The remains of the Shang culture sites distributed in the lakeside areas of Yangjiazui, Lijiazui, Xiaozui and Wangjiazui will no longer be submerged in normal season. The ramparts in the Panlongcheng site are no longer surrounded by lake water. Additionally, the finding of relics outside the ramparts would be reasonable.

Therefore, the land area in the Panlongcheng site in the Shang Dynasty should be large and contiguous rather than the broken shape of lake branches and low hills crisscrossing in modern times. Moreover, this is due to the Fu River joining the Yangtze River in Wuhan, which provided an important channel for the Panlongcheng site to communicate with the Central Plains culture and the upper and lower reaches of the Yangtze River, providing an important transportation condition for Panlongcheng city from an ordinary settlement in the late Xia Dynasty to a big city in the early Shang Dynasty.

In short, the restoration of landscapes and analysis of water-land distribution in the Panlongcheng site provides a new perspective to figure out the rise of Panlongcheng culture in the early Shang Dynasty and the strategic intention of the Shang Dynasty for the middle reaches of the Yangtze River.

\subsection{Disscusion and Future Work}

In this paper, a new method is proposed to restore the geomorphic distribution and water-land relationship distribution on a large scale near water sites. The upper soil is stripped by combining a large number of land and underwater archaeological hole data, so as to restore the geomorphology of the site in the period, thus analyzing the relationship between geomorphic changes and natural environment and human activities. Compared with traditional remote sensing methods, satellite images can not realize the perspective and restoration of underground landform. However, the GPR technology rising this year has high requirements for the soil environment, and its application in large-scale soil sites is not ready at present. Therefore, terrain restoration through archaeological borehole data 
is appropriate and feasible. Taking the Panlongcheng site as an example, this paper realizes this method and obtains good results.

However, geomorphic change is a long-term and complex process, but the accurate restoration of historical geomorphology requires more data and methods. Therefore, the analysis of paleo-environmental data and archaeological pit data needs to be explored in future work.

Moreover, the excavation and archaeological work of the Panlongcheng site are still going on, the excavated part on land only accounts for $60 \%$ of the total area of the site. Therefore, the stripping surface obtained by interpolation is not accurate in the area without archaeological exploratory holes around the site. With the development of archaeological work, more accurate geomorphic restoration result will be carried out in a wider range of site areas in the future. In addition, in the step of removal of artificial features, on the one hand, completely removing the DLG information of features will cause a large amount of information loss, we retain part of DLG information of some features. For example, the Fu River levee was originally built on hillock land, thus it is not appropriate to completely remove DLG of the Fu River levee when removing artificial features. Clarifying the removal of artificial landform in DLG and retain the original landform information is also necessary.

Lastly, it is very important to establish relief texture mapping to assist in displaying the geomorphic changes and water level changes in the Panlongcheng site. Generating accurate relief mapping with texture requires a lot of aerial photography and ground image acquisition, which will be completed in future work.

\section{Conclusions}

In this paper, with the help of spatial information technology, the data of archaeological excavation, exploration and investigation were used to restore the landscape of the Panlongcheng site in different historical periods. Moreover, the difference between the ancient settlement landscape and modern site landscape was visually presented. Relationships between water-land distribution and environmental change and human activities were analyzed. From the perspective of archaeology, this paper draws two conclusions. First, the restoration of the settlement landscape of the Panlongcheng site in the Shang Dynasty was realized with multi-disciplinary technology, which is of great significance for studying the layout of archaeological sites and exploring character of the settlement. Secondly, through a long period of landscape change analysis, the profound impact of human activities and environmental changes on the landscape of ancient sites was revealed, which provided a new viewpoint in studying the complex relationship between human activities and geographical environment changes in this area.

Author Contributions: Conceptualization, J.L., Q.Z. and Q.H.; methodology, Q.H.; software, J.L.; validation, J.L., Q.Z. and Q.H.; formal analysis, J.L.; investigation, J.L.; resources, Q.Z.; data curation, J.L.; writing—original draft preparation, J.L.; writing—review and editing, Q.Z.; visualization, J.L.; supervision, Q.Z.; project administration, C.Z.; funding acquisition, C.Z. All authors have read and agreed to the published version of the manuscript.

Funding: This research was funded by National Key R\&D Program of China, [grant numbers 2020YFC1521900] and the National Social Science Fund of China [grant numbers 547 16ZDA146].

Institutional Review Board Statement: Not applicable.

Informed Consent Statement: Not applicable.

Data Availability Statement: Restrictions apply to the availability of these data. Data was obtained from Wuhan University and are available from the the corresponding author with the permission of Wuhan University.

Acknowledgments: We would like to acknowledge the suggestions given by reviewers and editor. We are also indebted to Hubei Provincial Institute of Cultural Relics and Archaeology and Panlongcheng Archaeological Museum for their cooperation. Without their help, we would not be able to complete the study. 
Conflicts of Interest: We declared that we have no conflict of interest to this work. We declare that we do not have any commercial or associative interest that represents a conflict of interest in connection with the work submitted.

\section{References}

1. Llobera, M. Exploring the topography of mind: GIS, social space and archaeology. Antiquity 1996, 70, 612-622. [CrossRef]

2. Arıkan, B. Don't abhor your neighbor for he is a pastoralist: The GIS-based modeling of the past human-environment interactions and landscape changes in the Wadi el-Hasa, west-central Jordan. J. Archaeol. Sci. 2012, 39, 2908-2920. [CrossRef]

3. Burg, M.B. Reconstructing "total" paleo-landscapes for archaeological investigation: An example from the central Netherlands. J. Archaeol. Sci. 2013, 40, 2308-2320. [CrossRef]

4. Kempf, M. The application of GIS and satellite imagery in archaeological land-use reconstruction: A predictive model? J. Archaeol. Sci. Rep. 2019, 25, 116-128. [CrossRef]

5. Xu, S. Development of Shang Culture in Middle Yangtze River Region Based on Case Study on Panlongcheng. Jianghan Archaeol. 2003, 86, e44.

6. Zhang, H.; Wang, H.; Zou, Q.; Chen, H.; Su, X.; Liao, H. Researching the Changing Settlement Landscape of Panlongcheng during the Shang Dynasty. Jianghan Archaeol. 2018, 158, 139-152.

7. Zou, Q. The Preliminary Research of Geographic Environment Changing of Panlongcheng Site. Res. Herit. Preserv. 2017, 2, 160-165.

8. Zou, Q. Changes in the Environment of Panlongcheng as Seen through its Drainage System. Jianghan Archaeol. 2018, 158, 155-163.

9. Zhang, C.; Fang, Q.; Li, Y.; Wan, L. Archaeology in Panlongcheng Site from 2012 to 2017: Ideas and Gains. Jianghan Archaeol. 2018, $158,3-13$.

10. Singh, H.; Adams, J.; Mindell, D.; Foley, B. Imaging Underwater for Archaeology. J. Field Archaeol. 2000, 27, 319-328.

11. Passaro, S.; Barra, M.; Saggiomo, R.; Di Giacomo, S.; Leotta, A.; Uhlen, H.; Mazzola, S. Multi-resolution morpho-bathymetric survey results at the Pozzuoli-Baia underwater archaeological site (Naples, Italy). J. Archaeol. Sci. 2013, 40, 1268-1278. [CrossRef]

12. Tryon, C.A.; Faith, J.T.; Peppe, D.J.; Keegan, W.F.; Keegan, K.N.; Jenkins, K.H.; Beverly, E.J. Sites on the landscape: Paleoenvironmental context of late Pleistocene archaeological sites from the Lake Victoria basin, equatorial East Africa. Quat. Int. 2014, 331, 20-30. [CrossRef]

13. Mccarthy, J.; Benjamin, J. Multi-image Photogrammetry for Underwater Archaeological Site Recording: An Accessible, DiverBased Approach. J. Marit. Archaeol. 2014, 9, 95-114. [CrossRef]

14. Wang, J.; Lu, B.; Zhang, Y.; Cao, J. Application of Multi-beam Sonar Water Column Image in Object Detection in Shallow Water. Hydrogr. Surv. Charting 2018, 38, 54-58.

15. Reed, S.; Petillot, Y.; Bell, J. An automatic approach to the detection and extraction of mine features in sidescan sonar. IEEE J. Ocean. Eng. 2003, 28, 90-105. [CrossRef]

16. Smith, C.J.; Banks, A.C.; Papadopoulou, K.N. Improving the quantitative estimation of trawling impacts from sidescan-sonar and underwater-video imagery. Ices J. Mar. Sci. 2007, 1692-1701. [CrossRef]

17. Westley, K.; Plets, R.; Quinn, R.; Mcgonigle, C.; Sacchetti, F.; Dale, M.; McNeary, R.; Clements, A. Optimising protocols for high-definition imaging of historic shipwrecks using multibeam echosounder. Archaeol. Anthropol. Sci. 2019, 11, 3629-3645. [CrossRef]

18. Yan, C. A study on the evolution and geographical position of ancient city sites in Danjiangkou Reservoir Area. Jianghan Archaeol. 1996, 93-97.

19. Zou, Q.; Yao, S.; Li, C.; Hu, Q.; Zhang, C. A Preliminary Report on the Underwater Exploration and Test Excavation of the Panlongcheng Archaeological Site. Jianghan Archaeol. 2018, 158, 78-87.

20. Wang, S.; Jiong, L.I.; Wang, J. Study on the Production Technology and Quality Control of DLG Data Based on Virtuo-Zo and Geoway. Geomat. Spat. Inf. Technol. 2017, 1, 218-220, 224.

21. Su, X.; Zou, Q.; Yao, S.; Li, C.; Zhu, S.; Zhang, C. Mapping Panlongcheng: NewWork on the Type-Site of the Early Shang Period (1500-1300 BC) in Hubei Province, China. Land 2021, 10, 1033. [CrossRef]

22. Tsai, V.J. Delaunay triangulations in TIN creation: An overview and a linear-time algorithm. Geogr. Inf. Syst. 1993, 7, 501-524 [CrossRef]

23. Shi, W.Z.; Wang, B.; Tian, Y. Accuracy Analysis of Digital Elevation Model Relating to Spatial Resolution and Terrain Slope by Bilinear Interpolation. Math. Geosci. 2014, 46, 445-481. [CrossRef]

24. Du, J. A Discussion of the Shang Period Palace Foundation at Panlongcheng. Chin. Archaeol. 2006, 6, 140-146.

25. Wang, H.; Xiong, Y.; Zhang, J.; Li, Y.; Zuo, Y.; Xu, M. Assessment of schistosomiasis transmission risk after flood damage in Wuhan City. Chin. J. Schistosomiasis Control 2018, 30, 410-414. 